\title{
Phrase Searching for Encrypted Cloud Storage
}

\author{
${ }^{*}$ Ankita J. Gaware, ${ }^{2}$ Deepti. P. Theng \\ ${ }^{1,2}$ Computer Science and Engineering G. H. Raisoni College of Engineering \\ Email: ankitagaware999@gmail.com
}

Received: 09 ${ }^{\text {th }}$ July 2018, Accepted: $14^{\text {th }}$ August 2018, Published: $3^{\text {st }}$ August 2018

\begin{abstract}
The storage and access of confidential documents are known together for the central issues within the space. Whereas several schemes are planned to perform a conjunctive keyword search, less attention has been noted on more specialized looking techniques. There are several issues like as organizations and people adopt cloud technologies, several became aware of the intense considerations concerning security and privacy of accessing personal and confidential information over the net and conjointly there's want of correct looking. In existing conjunctive key search has less attention towards specialized search. In this paper, we tend to propose a phrase search theme that takes advantage of the house potency of Bloom filters. It makes use of bilaterally symmetrical cryptography, that provides process and storage potency over schemes supported public key cryptography. The theme provides the straightforward ranking capability, is custom-made to non-keyword search and is appropriate against inclusion-relation attack. Lastly, we tend to analyze our theme and value however it performs once changes.
\end{abstract}

Keywords: Cryptography, Cloud, Encryption Technique, Phrase, Hash function.

\section{Introduction}

Cloud computing empowers cloud clients to remotely store their information into the cloud in order to appreciate the on-request top notch applications and administrations from a common pool of configurable figuring assets. The benefits brought by this new processing model incorporate however are not constrained to: alleviation of the weight for capacity administration, all-inclusive information access with autonomous land areas, and evasion of capital use on equipment, programming, and faculty systems of support, etc.[2]. Cloud computing gives for all intents and purposes boundless computational and capacity assets and has pulled in expanding number of people and organizations to move their information into the cloud.

Long phrase questions are frequently used to find known things instead of to find assets for a general point. Much of the time, the objective is to distinguish a solitary archive. Longer phrase likewise have a low likelihood of event and yield less matches. Therefore, even with an exactness rate of half, we would infrequently observe more than a solitary false positive for a hunt inquiry of longer phrase [1]. The conversion rate conjointly will increase as results of your additional probably to own what the user is craving for. Rather like a keyword may be a single word used as a quest question, a keyword phrase is two or additional words typewritten as a quest question Users notice what they're craving for by sorting out specific keywords or keyword phrases and selecting the foremost relevant result.

Cloud Computing permits cloud customers to remotely store their information into the cloud thus on get pleasure from the on-demand prime quality applications and services from a shared pool of configurable computing resources. the advantages brought by this new computing model embody however aren't restricted to the relief of the burden for storage management, universal information access with freelance geographical locations, and rejection of cost on hardware, software, and personnel maintenances, etc. In paper [13] we've more studied the matter of searchable encoding, that solves the perplexity of maintaining the confidentiality of knowledge and therefore the ability for a consumer to go looking. We first introduce the model of phrase search with bilateral encoding and its security definition, and then propose a construction and its security proof. Lastly, we analyze our theme and measure however it performs once change [5].

\section{Related Work:}

In paper [1] conferred a phrase search theme supported Bloom filter that's considerably quicker than existing approaches, requiring solely one spherical of communication and Bloom filter verifications. The answer addresses the high process value noted in by reformulating phrase. Their approach is also the primary to effectively permit phrase search to run severally while not first playacting a conjunctive keyword search to spot candidate documents. The technique of constructing a Bloom filter index introduced allows quick verification of Bloom filters in the same manner as compartmentalization [6]. Strengths- scale back storage value and provide security within the sort of false positives and adapt the theme to defend against 
inclusion relation attacks. Weakness- The verification speed is less and fewer communication value.

In paper [7] they proposed a viable way to deal with take care of the issue of equivalent word based multi watchword positioned seek over encoded cloud information. The filed records can be refined when affirmed cloud customers input the comparable expressions of the predefined catchphrases, not the right or cushy organizing watchwords, due to the possible proportionate word substitution and also her nonappearance of right finding out about the data. For the first time they formalize and manage the issue of supporting efficient yet security ensuring padded look for accomplishing productive use of remotely set away blended information in Cloud Computing. Strengths- Computation complexness is greatly reduced and improves the potency of the server to retrieve the encryption information. Weakness-The server cannot generate trapdoor itself.

In paper [8] they have format an induced approach to gather the breaking point efficient delicate catchphrase sets by mauling a significant wisdom on the comparability metric of progress divided. In context of the created padded watchword sets, they have additionally propose an efficient cushy catchphrase look design. Through cautious security examination, they show that our proposed strategy is secure and confirmation guarding, while effectively understanding the objective of cushy catchphrase look.

In paper [9] they proposed a multi-catchphrase look plot in light of Wang et al's. conspire. They additionally novel technique for watchword change and present the stemming calculation. Their plan does not require a predefined catchphrase set and thus empowers efficient file refresh. In this paper, they examine the issue of multi-catchphrase cushioned situated investigate mixed cloud data. they propose a multi-catchphrase soft situated look for plan in perspective of Wang et al's. plot. Weakness-These schemes aim solely to protect the keyword set of a single question, whereas the relations between different queries don't seem to be studied. In paper [10] additional studied the matter of searchable encoding, that solves the perplexity of maintaining the confidentiality of knowledge and also the ability for a consumer to search. They have introduced the model of phrase search with symmetric encoding and its security definition, and then propose a construction and its security proof. They have proved that their scheme achieves non-adaptive security. StrengthsAchieves non accommodative security beneath the protection. Weakness- It doesn't meet the standards of adaptive security.
In paper [6] conferred a phrase search theme based mostly on Bloom filter that achieves eight times lower storage value in their experiment than the prevailing solutions whereas exhibiting similar or higher communication and process requirements. The planned resolution provides the basic ranking capability, may be custom-made to non-keyword search and is appropriate against inclusion-relation attacks[11]. Strengths- the flexibility to look over the encrypted information and provides the basic ranking capability, may be custom-made to non-keyword search and is appropriate against inclusion relation attack. Weakness- totally different split values may leak information on the document content.

In paper [14] they asked about the issue of articulation analyze mixed data and proposed a dynamic multiphrase orchestrated scan for over encoded data with symmetric open encryption. Not the same as prior work, our arrangement enables data customers to look through a couple of articulations in a demand request, and the data proprietor can vitalize the outsourced data at less cost. Remembering the true objective to rank the rundown things, they found the centrality scores inside the TFIDF appear on client side. It conceivably keeps up a key division from the spillage of significance scores. The novel synopsis associates with data customers to look encoded data successfully.

In paper [15] they propose another MRSE structure which beats each and every one of the bits of the KNN-SE based MRSE systems. Specifically, their new system does not require a predefined watchword set and sponsorships catchphrases in subjective tongues, is a multi-customer structure which reinforces flexible request guaranteeing and timecontrolled foreswearing, and it achieves better data security attestation since even the cloud server can't tell which records are the best $\mathrm{k}$ occurs obviously returned to a data customer. They proposed multicatchphrase rank open encryption which vanquishes every last one of the defects of the KNN-SE based MRSE frameworks

In paper[17] they proposed multi-keyword rank searchable encryption which conquers every one of the imperfections of the KNN-SE based MRSE frameworks. The framework permits flexible hunt approval and time controlled disavowal. They demonstrated the security of the framework and directed broad PC re-enactments to show its efficiency. Strengths- The system allows flexible search authorization and time-controlled revocation. In paper [16] they stick and handle the issue of secure multi-watchword top-k recovery over blended cloud information. They defined respectability congruity and plan quality. In light of order preserving encryption bafflingly release scrappy data, they 
devise a server side planning SSE make. They by then propose a two-round open encryption (TRSE) plot utilizing the absolutely homomorphic encryption, which fulfills the security stray bits of multiwatchword top recovery over the encoded cloud information. By security examination, they show that the proposed plot ensures information inquire. As showed up by the efficiency appraisal of the proposed devise over declared dataset, wide test works out unmistakably exhibit that our framework guarantees sensible efficiency.

\section{Proposed System:}

To overcome the matter i.e. organizations and people adopt cloud technologies, several became aware of the intense considerations relating to security and privacy of accessing personal and Confidential information over the web, conjointly there's would like of correct looking out. In existing conjunctive key search has less attention towards specialized look for that purpose we have designed quick verifications, to produce quick search over encrypted cloud storage conjointly provide the specialized looking out technique.[12] When no-hit of this research will be used in search engines, looking sites, permits quick verifications, Achieves low storage price, Maximize looking out speed with affordable storage, offer defense against inclusion relation -attack. We have to commit for executing the given work by including phrase keyword search then produce the cloud within which knowledge is going to be stored within the encrypted type, then the user can send the question request to the admin. The admin or the info owner can search phrase keyword search victimization n-grams series to take apart the keywords within the encrypted information. Using encryption algorithm for encrypting the data to store on the cloud In the encrypted information, the info is going to be decrypted for looking out the keyword victimization Hash perform. After finding the correct result it'll be forwarded to the actual user. For executing the system we have to work on the three contents i.e. remote cloud for knowledge storage; knowledge owner to transfer knowledge with encrypted type and last is knowledge scientist to look for encrypted knowledge.

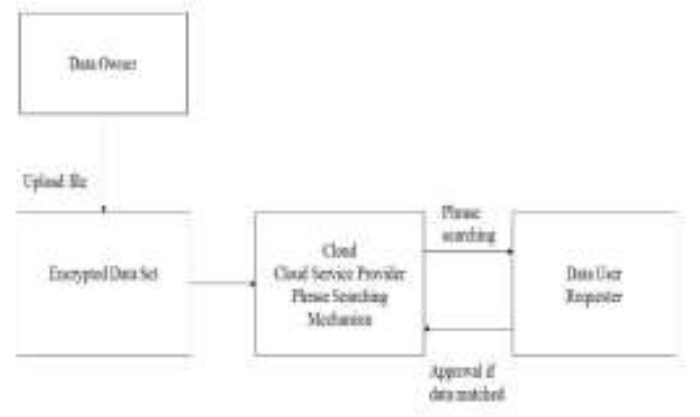

\section{Fig: Working of System}

Steps of project designing work and as the responsibility of employee what we've to try and do. A. As knowledge owner- the info homeowners will be relieved from the burden of information storage and maintenance therefore as to relish the on-demand prime quality knowledge storage service. However, the very fact that knowledge homeowners and cloud server aren't in the same trustworthy domain might place the outsourced knowledge in danger, as the cloud server might not be absolutely trustworthy.[14] Wherever the info uploads the documents on a cloud with secret writing. For those encrypting documents, we have a tendency to area unit victimization hash perform with a set of keywords. And no matter set of keywords and phrases are going to be connected to knowledge owner.

B. Owner store the info on a cloud with several storage houses- here the cloud service supplier provides the space likewise because the third party receives in terms of authentication. Cloud service supplier receives scientist request with a set of phrase.

C. Knowledge or documents searcher- Here the question part is generated for documents to be searched. Cloud service supplier can receive the request.

\section{Conclusion}

In this paper, we presented different kinds of literature based on the Enhanced Phrase Searching Mechanism for Encrypted Cloud Storage and we have planned some steps to follow by owner. We have to first create the cloud or own the space in it within which knowledge is going to be stored in encrypted form. The user can send request to the admin. Else user can directly search for the file. In these way the organizations and people adopt can use cloud technologies, several became aware of the intense considerations relating to security and privacy of accessing personal and confidential information over the web. In this paper, we've got more studied the matter of searchable cryptography, that solves the quandary of maintaining the confidentiality of information and also the ability for a consumer to look. We tend to initially introduce the model of phrase search with bilaterally symmetrical cryptography and its security definition, then propose a construction and its security proof

\section{References}

1. H. T. Poon and A. Miri, "Fast Phrase Search for Encrypted Cloud Storage," vol. 7161, no. c, pp. 112, 2017. 
2. C. Wang, N. Cao, J. Li, K. Ren, and W. Lou, "Secure Ranked Keyword Search over Encrypted Cloud Data," 2010

3. Y. Fu, N. Xiao, H. Jiang, G. Hu, and W. Chen, "Application-Aware Big Data Deduplication in Cloud Environment," vol. 7161, no. c, pp. 1-14, 2017.

4. Z. Yan, S. Member, X. Li, M. Wang, and A. V Vasilakos, "Flexible Data Access Control based on Trust and Reputation in Cloud Computing," vol. 7161, no. c, 2015.

5. H. T. Poon and A. Miri, "A Low Storage Phase Search Scheme based on Bloom Filters for Encrypted Cloud Services,'2015.

6. M. Ding, F. Gao, Z. Jin, and H. Zhang, "An Efficient Public Key Encryption With Conjunctive Keyword Search Scheme Based,” pp. 526-530, 2012.

7. J. Li, Q. Wang, C. Wang, N. Cao, K. Ren, and W. Lou, "Fuzzy Keyword Search over Encrypted Data in Cloud Computing,"2010.

8. Y. Tang, D. Gu, N. Ding, and H. Lu, "Phrase Search over Encrypted Data with Symmetric Encryption Scheme," 2012.

9. M. A. Chauhan and C. W. Probst, "Architecturally Significant Requirements Identification, Classification and Change Management for Multi-tenant Cloud-Based Systems," 2017.

10. Chen R, Mu Y, Yang G, et al. Dual-server publickey encryption with keyword search for secure cloud storage[J]. IEEE Transactions on Information Forensics and Security, , 11(4): 789-798. 2016.

11. Fu Z, Sun X, Linge N, et al. Achieving effective cloud search services: multi-keyword ranked search over encrypted cloud data supporting synonym query[J]. IEEE Transactions on Consumer Electronics, 60(1): 164172. 2014

12. $\mathrm{Yu}$ J, Lu P, Zhu Y, et al. Toward secure multi keyword top-k retrieval over encrypted cloud data[J]. IEEE transactions on dependable and secure computing, , 10(4): 239-250, 2013

13. Cong Wang et al.," Enabling Secure and Efficient Ranked Keyword Search over Outsourced Cloud Data", IEEE Transactions on parallel and distributed systems, vol. 23, no.8, August 2012

14. Zhihua Xia, "A Secure and Dynamic Multikeyword Ranked Search Scheme over Encrypted
Cloud Data", IEEE Transactions On Parallel And Distributed Systems, Vol: Pp No: 99 Year 2015

15. Z. J. Fu, X. L. Wu, C. W. Guan, X. M. Sun, and K. Ren, "Toward Efficient Multi-keyword Fuzzy Search over Encrypted Outsourced Data with Accuracy Improvement," IEEE Transactions on Information Forensics and Security, vol. 11, no. 12, pp. 2706-2716, Dec. 2016

16. Cheng Guo, Xue Chen, Yingmo Jie, Zhangjie Fu, Mingchu Li, and Bin Feng, "Dynamic Multi-phrase Ranked Search over Encrypted Data with Symmetric Searchable Encryption",IEEE Transactions on Services Computing, .2768045, 2017

17. Yang Yang, Ximeng Liu, Robert H. Deng," Multiuser Multi-Keyword Rank Search over Encrypted Data in Arbitrary Language", IEEE Tranactions on Dependable and Secure Computing, 2787588, 2017. 